Binding energies and external-field effects of the A-exciton states in CdS

This content has been downloaded from IOPscience. Please scroll down to see the full text. 1990 J. Phys.: Condens. Matter 22807

(http://iopscience.iop.org/0953-8984/2/12/004)

View the table of contents for this issue, or go to the journal homepage for more

Download details:

IP Address: 140.113.38.11

This content was downloaded on 28/04/2014 at 19:51

Please note that terms and conditions apply. 


\title{
Binding energies and external-field effects of the A-exciton states in CdS
}

\author{
C M Dai†, J H Peił, W N Mei§ and D S Chuul| \\ $\dagger$ Institute of Electro-Optics, National Chiao Tung University, Hsinchu, Taiwan, \\ Republic of China \\ $\ddagger$ Institute of Electronics, National Chiao Tung University, Hsinchu, Taiwan, \\ Republic of China \\ $\S$ Department of Physics, University of Nebraska, Omaha, Nebraska 68182, USA \\ II Department of Electrophysics, National Chiao Tung University, Hsinchu, Taiwan, \\ Republic of China
}

Received 1 June 1989, in final form 12 August 1989

\begin{abstract}
Based on the effective-mass approximation, the energy spectrum of the free Aexciton states of cadmium sulphide is studied. The Stark effect in an electric field, the Zeeman effect and the diamagnetic interaction in a magnetic field of the A-exciton states are analysed by using a novel perturbative-variational approach. The results are compared with experimental data and previous calculations using different approaches. Our results are in good agreement with previous work and our method is much more efficient.
\end{abstract}

\section{Introduction}

It is known that cadmium sulphide has a hexagonal wurtzite structure. Its space group is $\mathrm{C}_{6 \mathrm{v}}^{4}$, with the hexagonal axis generally referred to as the $c$ axis. This structural anisotropy causes crystal-field splitting, which interacts with the spin-orbit coupling to split the valence band into three nearly degenerate sub-bands, A, B and C. The lowest band gap is due to the A valence band and is at the Brillouin zone centre. The band-gap energy of the A valence band, which is a direct band, was measured by Seiler et al (1982) to be about $2.5825 \mathrm{eV}$ at $1.8 \mathrm{~K}$. The energy splitting of the valence band has been considered by Thomas and Hopfield (1961) and Litton et al (1972). It is usually believed that the $A$ and $B$ valence bands are split by the crystal field with a value of $11-16 \mathrm{meV}$ and that the $C$ band is split by the spin-orbit interaction with a value around $57-109 \mathrm{meV}$ below the $\mathrm{B}$ band.

Over the past 30 years, numerous studies have been performed and detailed information about the nature of the free A-excitons in CdS and their behaviour in a magnetic field have been provided. Linear spectroscopy using one-photon reflectance (Thomas and Hopfield 1959, Blattnor et al 1982, Venghaus et al 1977, Broser and Rosenzweig 1980), one-photon absorption (Hopfield and Thomas 1961, Blattnor et al 1982, Shad and Damen 1971, Thomas and Hopfield 1961, Peyghambarian et al 1989) and onephoton emission or luminescence (Litton et al 1972, Thomas and Hopfield 1959, Cho et al 1975, Suga et al 1975, Venghaus et al 1977, Broser and Rosenzweig 1980) and nonlinear spectroscopy using two-photon absorption (Seiler et al 1982, Nquyen et al 1977a,b, 
Stafford and Sondergeld 1974, Damen et al 1977, Seiler et al 1983) techniques allowed various features of the magneto-optical effects in the A-exciton spectrum to be investigated. The primary features were Zeeman splittings and diamagnetic shifts, from which effective masses and $g$-factors for conduction band and A valence band were extracted.

Many different approaches based on the effective-mass approximation have been used to interpret the experimental data. Seiler et al (1982) and Hopfield and Thomas (1961) used a model semiconductor with conduction and simple A valence bands. In the work of Hopfield and Thomas (1961), a small anisotropy parameter $\gamma$ was introduced and the traditional perturbation approach was performed. A hydrogenic wavefunction was assumed as the unperturbed wavefunction; excellent interpretation of the observed data at zero external field was obtained. Seiler et al (1982) used a variational method with a hydrogen-like trial wavefunction containing four or five parameters. Good agreement is obtained. Many others (Cho et al 1975, Blattnor et al 1982, Suga et al 1975, Venghaus et al 1977, Voigt et al 1979, Broser and Rosenzweig 1980, Pollmann 1976, Gerlach and Pollmann 1977, Seiler et al 1982, Grundmann and Bimberg 1988) have considered the mixing and splitting of $\mathrm{A}$ and $\mathrm{B}$ valence bands under the interaction of electric and magnetic fields and large anisotropic mass effects. In these previous works, three different types of effects are usually included: namely, (i) the electron-hole exchange effect; (ii) the linear Zeeman effect; and (iii) the diamagnetic interactions. Under these circumstances, the Hamiltonian is in general an $8 \times 8$ matrix. Lipari (1972) took into account the coupling of $\mathrm{A}, \mathrm{B}$ and $\mathrm{C}$ valence bands and, neglecting the exchange effect due to the magnetic field, simplified the Hamiltonian into a $6 \times 6$ matrix.

In the present work, we propose a simple but much more efficient approximation method to study the binding energies and electric and magnetic splittings of the exciton states in CdS. For the purpose of illustration, we considered only the A valence band. Since the coupling between the A- and B-exciton levels is small in the weak-field region, we believe our treatment should be reasonable there. More exploratory work containing the mixing between $\mathrm{A}$ and $\mathrm{B}$ bands is in progress and the results will be published soon. Our calculation is based on the effective-mass approximation. The approximation method used in the present work combines the spirit of both the conventional perturbative and the variational methods and was originally proposed by Lee et al (1982). The main idea of this perturbative-variational method (denoted PVM) is that, if a Hamiltonian that is originally not exactly solvable is added to and subtracted from the same term of some parameter $\lambda$, the new Hamiltonian can be separated into two terms, with one term being exactly solvable while the other can be treated as a perturbation. Then the perturbation series of the energy eigenvalue can be made to converge rapidly by requiring the value of $\lambda$ to be chosen such that the first-order energy correction is as small as possible. This method has been successfully applied to the impurity-level calculations of both isotropic and anisotropic semiconductors (Jiang and Shan 1985, Huang et al 1988). Good agreement has been obtained.

\section{Theory}

\subsection{Free-exciton binding energies}

The energy level spectrum of the free A-excitons of CdS can be conveniently calculated within the framework of the effective-mass approximation. The basic Hamiltonian appropriate to our case (Hopfield and Thomas 1961) can be expressed as follows: 


$$
\begin{aligned}
H=-\frac{\hbar^{2}}{2 m_{\mathrm{e} \perp}} & \left(\frac{\partial^{2}}{\partial x_{\mathrm{e}}^{2}}+\frac{\partial^{2}}{\partial y_{\mathrm{e}}^{2}}\right)-\frac{\hbar^{2}}{2 m_{\mathrm{e} \|}} \frac{\partial^{2}}{\partial z_{\mathrm{e}}^{2}}-\frac{\hbar^{2}}{2 m_{\mathrm{h} \perp}}\left(\frac{\partial^{2}}{\partial x_{\mathrm{h}}^{2}}+\frac{\partial^{2}}{\partial y_{\mathrm{h}}^{2}}\right)-\frac{\hbar^{2}}{2 m_{\mathrm{h} \|}} \frac{\partial^{2}}{\partial z_{\mathrm{h}}^{2}} \\
& -e^{2} /\left[\varepsilon_{\perp} \varepsilon_{\|}\left(x_{\mathrm{e}}-x_{\mathrm{h}}\right)^{2}+\varepsilon_{\perp} \varepsilon_{\|}\left(y_{\mathrm{e}}-y_{\mathrm{h}}\right)^{2}+\varepsilon_{\perp}^{2}\left(z_{\mathrm{e}}-z_{\mathrm{h}}\right)^{2}\right]^{1 / 2}
\end{aligned}
$$

where the $z$ axis is chosen in the $c$ direction of the hexagonal structure and the static anisotropic dielectric constant is assumed. Here $m_{\mathrm{e} \perp}\left(m_{\mathrm{h} \perp}\right)$ is the electron (hole) effective mass perpendicular to the $c$ axis; $m_{\mathrm{e} \|}\left(m_{\mathrm{h} \|}\right)$ is the electron (hole) effective mass parallel to the $c$ axis; $x_{\mathrm{e}}, y_{\mathrm{e}}, z_{\mathrm{e}}\left(x_{\mathrm{h}}, y_{\mathrm{h}}, z_{\mathrm{h}}\right)$ are the electron (hole) coordinates; and $\varepsilon_{\perp}\left(\varepsilon_{\|}\right)$is the low-frequency (clamped) dielectric constant perpendicular (parallel) to the $c$ axis. The Hamiltonian defined in equation (1) can be reduced to a simpler form by considering some simple transformations. Use a centre-of-mass coordinate system where the centreof-mass motion is set to zero and perform the following transformation:

$$
\begin{array}{ll}
x=x_{\mathrm{e}}-x_{\mathrm{h}} \quad y=y_{\mathrm{e}}-y_{\mathrm{h}} \quad z=\left(\varepsilon_{\perp} / \varepsilon_{\|}\right)^{1 / 2}\left(z_{\mathrm{e}}-z_{\mathrm{h}}\right) \quad \varepsilon_{0}=\left(\varepsilon_{\|} / \varepsilon_{\perp}\right)^{1 / 2} \\
\frac{1}{\mu_{\perp}}=\frac{1}{m_{\mathrm{e} \perp}}+\frac{1}{m_{\mathrm{h} \perp}} \quad \frac{1}{\mu_{\|}}=\frac{1}{m_{\mathrm{e} \|}}+\frac{1}{m_{\mathrm{h} \|}} \\
\frac{1}{\mu_{0}}=\frac{2}{3} \frac{1}{\mu_{\perp}}+\frac{1}{3} \frac{1}{\mu_{\|}} \frac{\varepsilon_{\perp}}{\varepsilon_{\|}} \quad \gamma=\mu_{0}\left(\frac{1}{\mu_{\perp}}-\frac{1}{\mu_{\|}} \frac{\varepsilon_{\perp}}{\varepsilon_{\|}}\right) .
\end{array}
$$

Here $\varepsilon_{0}$ is the effective static dielectric constant, $\mu_{0}$ is the effective mass of the electron and $\gamma$ is the anisotropy parameter of the exciton level. Then equation (1) can be rewritten as

$$
H=-\frac{\hbar^{2}}{2 \mu_{0}} \nabla^{2}-\frac{\hbar^{2}}{6 \mu_{0}} \gamma \nabla^{2}+\frac{\hbar^{2}}{2 \mu_{0}} \gamma \frac{\partial^{2}}{\partial z^{2}}-\frac{e^{2}}{\varepsilon_{0} r} .
$$

Originally, Hopfield and Thomas (1961) treated the second and third terms in equation (2) as the perturbation. This process requires tedious calculation of a large number of matrix elements when we extend it to higher-order corrections. Now, if we regroup the terms in equation $(2 a)$, the Hamiltonian can be changed into the following form:

$$
H=-\frac{\hbar^{2}}{2 \mu_{\mathrm{t}}}\left(\frac{\partial^{2}}{\partial x^{2}}+\frac{\partial^{2}}{\partial y^{2}}\right)-\frac{\hbar^{2}}{2 \mu_{1}} \frac{\partial^{2}}{\partial z^{2}}-\frac{e^{2}}{\varepsilon_{0} r}
$$

where

$$
1 / \mu_{\mathrm{t}}=1 / \mu_{0}+\gamma^{\prime} / 3 \quad 1 / \mu_{1}=1 / \mu_{0}-2 \gamma^{\prime} / 3 \quad \gamma^{\prime}=\gamma / \mu_{0}
$$

This Hamiltonian looks exactly the same as that of the anisotropic semiconductor with transverse mass $\mu_{\mathrm{t}}$ and longitudinal mass $\mu_{1}$ (Lee et al 1982). In fact, we found that Hopfield's perturbative result is slightly lower than that obtained by Kohn and Luttinger (1955). Thus, it seems to indicate the inaccuracy in treating those two terms as a perturbation.

The Hamiltonian in equation $(2 b)$ can be reduced further into a simpler form by considering the following transformation:

$$
x^{\prime}=x \quad y^{\prime}=y \quad z^{\prime}=\left(\mu_{1} / \mu_{\mathfrak{t}}\right)^{1 / 2} z
$$

Then equation $(2 b)$ can be rewritten as

$$
H=-\frac{\hbar^{2}}{2 \mu_{\mathrm{t}}} \nabla^{\prime 2}-\frac{e^{2}}{\varepsilon_{0} r^{\prime}} \frac{1}{\left(1-\Delta^{2} \cos ^{2} \theta\right)^{1 / 2}}
$$

where $\Delta^{2}=1-\mu_{\mathrm{t}} / \mu_{1}$. To solve the problem, we introduce a parameter $\lambda$ into $H$ by 
adding and subtracting a term $\lambda e^{2} / \varepsilon_{0} r$ to make the Hamiltonian $H$ separate into two parts (drop the prime for simplicity):

$$
H=H_{0}(\lambda)+H^{\prime}(\lambda)
$$

where the unperturbed part $H_{0}$ and the perturbation $H^{\prime}(\lambda)$ are

$$
\begin{aligned}
& H_{0}(\lambda)=-\frac{\hbar^{2}}{2 \mu_{\mathrm{t}}} \nabla^{2}-\frac{\lambda e^{2}}{\varepsilon_{0} r} \\
& H^{\prime}(\lambda)=\frac{e^{2}}{\varepsilon_{0} r}\left(\lambda-\frac{1}{\left(1-\Delta^{2} \cos ^{2} \theta\right)^{1 / 2}}\right) .
\end{aligned}
$$

The solutions for $H_{0}$ are the well known hydrogenic wavefunction

$$
\Psi_{n l m}(\rho, \theta, \varphi)=R_{n l}(\rho) Y_{l m}(\theta, \varphi)
$$

with the unperturbed eigenvalues expressed in units of mean rydbergs (Ryd) as

$$
E_{n l m}^{0}(\lambda)=-\lambda^{2} / n^{2}
$$

where $1 \mathrm{Ryd}=e^{2} /\left(2 a_{0}^{*} \varepsilon_{0}\right)$, and the effective Bohr radius $a_{0}^{*} \equiv \varepsilon_{0} \hbar^{2} /\left(\mu_{\mathrm{t}} e^{2}\right)$.

The first-order energy matrix correction can be evaluated by the conventional perturbation method, the diagonal terms for the $n l m$ state being expressed (in Ryd) as

$$
\Delta E_{n l m}^{(1)}(\lambda)=\frac{2 \lambda^{2}}{n^{2}}-\frac{2 \lambda}{n^{2}} S_{l m}
$$

where

$$
S_{l m}=\left\langle\operatorname{lm}\left|\frac{1}{\left(1-\Delta^{2} \cos ^{2} \theta\right)^{1 / 2}}\right| \operatorname{lm}\right\rangle .
$$

The values of $S_{l m}$ for some $l$ and $m$ and the non-zero off-diagonal matrix elements of the first-order energy correction are listed in appendix 1 and appendix 2 , respectively. To obtain the total energy for the whole Hamiltonian, we first have to determine the value of $\lambda$. For this purpose, we will employ a novel perturbative-variational method (PVM) (Lee et al 1982) to determine the parameter $\lambda$. The main idea of this method is to find a suitable $\lambda$ that will cause the perturbation series

$$
\begin{gathered}
E_{n l m}=E_{n l m}^{0}(\lambda)+\Delta E_{n l m}^{(1)}(\lambda)+\Delta E_{n l m}^{(2)}(\lambda)+\ldots \\
=E_{n l m}^{0}(\lambda)+\Delta E_{n l m}(\lambda)
\end{gathered}
$$

to converge as fast as possible so that only the first few terms will be needed. Hence $\lambda$ is to be determined by the condition

$$
\left|\frac{\Delta E_{n l m}(\lambda)}{E_{n l m}^{(0)}(\lambda)}\right|=\text { minimum }
$$

which can be satisfied by requiring that

$$
\Delta E_{n l m}(\lambda)=0 .
$$

If $\Delta E_{n l m}(\lambda)$ is approximated by $\Delta E_{n l m}^{(1)}(\lambda)$ of equation (8), the condition of equation (10) can be satisfied by requiring $\Delta E_{n l m}^{(1)}(\lambda)=0$, using equations $(6 b)$ and (10), yielding

$$
\lambda=S_{l m}
$$

and the total energy eigenvalues are thus

$$
E_{n l m}(\lambda)=\left(-1 / n^{2}\right) S_{l m}^{2}
$$




\subsection{The orbital magnetic effect: $\boldsymbol{B} / / \mathrm{c}$ axis}

When the direction of the external magnetic field is parallel to the hexagonal axis, the dominant two magnetic perturbations (Knox 1963) are: (i) the ordinary Zeeman term, which can be expressed in spherical coordinates as $i \mu_{\mathrm{B}} g_{\mu \|} B \partial / \partial \varphi$, where $\mu_{\mathrm{B}}=e \hbar / 2 m c$ is the Bohr magneton and $g_{\mu \|}=\left(1 / m_{\mathrm{h} \|}-1 / m_{\mathrm{e} \|}\right) m$; and (ii) the ordinary diamagnetic term $\left(C B^{2} / a_{0}^{* 2}\right)\left(x^{2}+y^{2}\right)$, where $C=e^{2} \hbar^{2} a_{0}^{* 2} / 8 \mu_{\perp} c^{2}$ and $a_{0}^{*}=\varepsilon_{0} \hbar^{2} / \mu_{\mathrm{t}} e^{2}$ is the effective Bohr radius. Therefore, the total Hamiltonian is

$H=-\frac{\hbar^{2}}{2 \mu_{\mathrm{t}}} \nabla^{\prime 2}-\frac{e^{2}}{\varepsilon_{0} r^{\prime}} \frac{1}{\left(1-\Delta^{2} \cos ^{2} \theta\right)^{1 / 2}}+\mathrm{i} \mu_{\mathrm{B}} g_{\mu \|} B \frac{\partial}{\partial \varphi}+\frac{C B^{2}}{a_{0}^{2}}\left(x^{2}+y^{2}\right)$.

The Schrödinger equation $H \Psi=E \Psi$ can be solved by the perturbative-variational approach as mentioned in the last section by introducing a term $\lambda e^{2} / \varepsilon_{0} r$ to make the Hamiltonian $H$ separate into two parts:

$$
H=H_{0}(\lambda)+H^{\prime}(\lambda) \text {. }
$$

The unperturbed Hamiltonian is now expressed as

$$
H_{0}(\lambda)=-\frac{\hbar^{2}}{2 \mu_{\mathrm{t}}} \nabla^{2}-\frac{\lambda e^{2}}{\varepsilon_{0} r}
$$

and the perturbed part is

$H^{\prime}(\lambda)=\frac{e^{2}}{\varepsilon_{0} r}\left(\lambda-\frac{1}{\left(1-\Delta^{2} \cos ^{2} \theta\right)^{1 / 2}}\right)+\mathrm{i} \mu_{\mathrm{B}} g_{\mu} B \frac{\partial}{\partial \varphi}+\frac{C B^{2}}{a_{0}^{* 2}}\left(x^{2}+y^{2}\right)$.

The solutions for the unperturbed Hamiltonian $H_{0}$ are the hydrogenic wavefunctions with eigenvalues (in unit of mean rydbergs, $e^{2} / 2 a_{0}^{*} \varepsilon_{0}$ )

$$
E_{n l m}^{0}(\lambda)=-\lambda^{2} / n^{2}
$$

and the energy correction due to $H^{\prime}$ of the diagonal matrix elements can be expressed as

$$
\begin{aligned}
\Delta E_{n l m}^{(1)}(\lambda)= & 2 \lambda^{2} / n^{2}-\left(2 \lambda / n^{2}\right) S_{l m}-2 a_{0}^{*} m \varepsilon_{0} \mu_{\mathrm{B}} g_{\mu \|} B / e+n^{2}\left[5 n^{2}+1-3 l(l+1)\right] \\
& \times\left(2 l^{3}+3 l^{2}-l+2 l m^{2}+m^{2}-1\right) C^{\prime} B^{2} /(2 l-1)(2 l+1)(2 l+3) \lambda^{2}
\end{aligned}
$$

where $C^{\prime}=\hbar^{2} \varepsilon_{0} a_{0}^{* 3} /\left(4 \mu_{\perp} c^{2}\right)$. The eigenvalues of the total Hamiltonian $H$ can be obtained numerically by diagonalising $H$, and the parameter $\lambda$ can be obtained by PVM as mentioned in the last subsection.

\subsection{The orbital magnetic effect: $\boldsymbol{B} \perp \mathrm{c}$ axis and $\boldsymbol{B} \| x$ axis}

When the magnetic field is applied in the direction perpendicular to the $c$ axis and pointing in the $x$ direction, the perturbation term, which depends linearly on the magnetic field, will mix the non-degenerate states $2 \mathrm{p}_{0}$ and $2 \mathrm{p}_{ \pm 1}$. One can choose a gauge that is 
slightly asymmetric in such a way that the coupling by the linear term of $n=2$ states to $n=3$ states is minimised. In such a gauge, the perturbed term can be expressed as

$$
H^{\prime}(\lambda)=\frac{e^{2}}{\varepsilon_{0} r}\left(\lambda-\frac{1}{\left(1-\Delta^{2} \cos ^{2} \theta\right)^{1 / 2}}\right)+H_{\mathrm{B}}^{\prime}
$$

where

$$
H_{\mathrm{B}}^{\prime}=\frac{e B}{2 c}\left(\mu_{\perp} \mu_{\|}\right)^{1 / 2}\left(\frac{1}{m_{\mathrm{e} \perp} m_{\mathrm{e} \|}}-\frac{1}{m_{\mathrm{h} \perp} m_{\mathrm{h} \|}}\right) L_{x}
$$

and the diamagnetic terms are neglected because they are believed to be small enough. The matrix elements of $L_{x}$ can be expressed as

$$
\begin{aligned}
\left\langle l^{\prime} m^{\prime}\left|L_{x}\right| l m\right\rangle & =(\hbar / 2)[(l-m)(l+m+1)]^{1 / 2} \delta_{l l^{\prime}} \delta_{m^{\prime}, m+1} \\
& +(\hbar / 2)[(l+m)(l-m+1)]^{1 / 2} \delta_{l^{\prime}} \delta_{m^{\prime}, m-1} .
\end{aligned}
$$

The parameter $\lambda$ can be determined by PVM as we did in section 2.1 .

\subsection{The electric effect: $\boldsymbol{E} \| c$ axis}

When the electric field is applied in the direction parallel to the $c$ axis, then the dominant effect on the exciton, which is the bounded electron-hole pair, will be the dipole effect. Since the quadrupole effect is usually small compared with the dipole effect, it is usual to neglect it. The perturbed Hamiltonian in this case is

$$
\begin{aligned}
& H^{\prime}(\lambda)=\frac{e^{2}}{\varepsilon_{0} r}\left(\lambda-\frac{1}{\left(1-\Delta^{2} \cos ^{2} \theta\right)^{1 / 2}}\right)+H_{\mathrm{E}^{\prime}}^{\prime} \\
& H_{\mathrm{E}}^{\prime}=-e E\left(\varepsilon_{\|} / \varepsilon_{\perp}\right)^{1 / 2} z .
\end{aligned}
$$

The matrix elements of $z$ for $n^{\prime}=n$ can be evaluated as

$$
\begin{aligned}
\left\langle n l^{\prime} m^{\prime}|z| n l m\right\rangle & =\frac{3 n}{2 \lambda} a_{0}^{*}\left\{d_{l, m}\left[n^{2}-(l+1)^{2}\right]^{1 / 2} \delta_{l^{\prime}, l+1} \delta_{m^{\prime}, m}\right. \\
& \left.+d_{l-1, m}\left(n^{2}-l^{2}\right)^{1 / 2} \delta_{l^{\prime}, l-1} \delta_{m^{\prime}, m}\right\}
\end{aligned}
$$

where

$$
d_{l, m}=\left\{\left[(l+1)^{2}-m^{2}\right] /[(2 l+1)(2 l+3)]\right\}^{1 / 2} .
$$

For the matrix elements with $n^{\prime} \neq n$, one can use the following formula:

$$
\begin{aligned}
\left\langle n l|r| n^{\prime}, l-1\right\rangle & =\frac{(-1)^{n^{\prime}-l}}{4 \lambda(2 l-1) !} a_{0}^{*}\left(\frac{(n+l) !\left(n^{\prime}+l-1\right) !}{(n-l-1) !\left(n^{\prime}-l\right) !}\right)^{1 / 2} \frac{\left(n-n^{\prime}\right)^{n+n^{\prime}-2 l-2}}{\left(n+n^{\prime}\right)^{n^{\prime}+n}} \\
& \times\left(4 n n^{\prime}\right)^{l+1} F\left(-n_{r},-n_{r}^{\prime} ; 2 l ;-\frac{4 n n^{\prime}}{\left(n-n^{\prime}\right)^{2}}\right)-\left(\frac{n-n^{\prime}}{n+n^{\prime}}\right)^{2} \\
& \times F\left(-n_{r}-2,-n_{r}^{\prime} ; 2 l ;-\frac{4 n n^{\prime}}{\left(n-n^{\prime}\right)^{2}}\right)
\end{aligned}
$$

where $n_{r}=n-l-1, n_{r}^{\prime}=n^{\prime}-l$ and

$$
F(\alpha, \beta ; \gamma ; \chi)=1+\frac{\alpha \beta}{\gamma \cdot 1} \chi+\frac{\alpha(\alpha+1) \beta(\beta+1)}{\gamma(\gamma+1) \cdot 2 !} \chi^{2}+\ldots
$$

The parameter $\lambda$ can be determined by PVM as mentioned in section 2.1 . 
Table 1. Values of some physical quantities for CdS. The effective electron and hole masses are in terms of the electron mass. The data listed in the first and second rows are adopted from Hopfield and Thomas (1961) and Seiler et al (1982) (denoted HT and SL).

\begin{tabular}{lllllllll}
\hline Authors & $m_{\mathrm{e} \perp}$ & $m_{\mathrm{e} \|}$ & $\varepsilon_{\mathrm{H}}$ & $\varepsilon_{\perp}$ & $m_{\mathrm{h} \perp}$ & $m_{\mathrm{h} \|}$ & $\gamma$ & $\mu_{\perp}$ \\
\hline HT & 0.205 & 0.205 & 9.53 & 9.02 & 0.70 & 5.0 & 0.258 & 0.159 \\
SL & 0.210 & 0.210 & 8.90 & 8.90 & 0.64 & 0.64 & 0.2177 & 0.158 \\
\hline
\end{tabular}

Table 2. A-exciton energy levels referred to the band gap of $A$ valence band to conduction band $E_{\mathrm{g}}^{*}=2.5825 \mathrm{eV}$ (at $1.8 \mathrm{~K}$ ) of $\mathrm{CdS}$. The energy is expressed in meV.

\begin{tabular}{lcccccc}
\hline & & \multicolumn{2}{c}{ Theor. } & & \multicolumn{2}{c}{ Theor. } \\
\cline { 3 - 5 } \cline { 5 - 6 } State & $\begin{array}{l}\text { Exp. } \\
(\mathrm{HT})\end{array}$ & Ours & HT & $\begin{array}{l}\text { Exp } \\
(\mathrm{SL})\end{array}$ & Ours & $\mathrm{sL}$ \\
\hline $1 \mathrm{~s}$ & 29.8 & 27.375 & 27.0 & - & - & - \\
$2 \mathrm{~s}$ & 6.9 & 6.844 & 6.7 & - & - & - \\
$2 \mathrm{p}_{0}$ & 7.5 & 7.345 & 7.1 & 7.86 & 7.744 & 8.247 \\
$2 \mathrm{p}_{ \pm 1}$ & 6.9 & 6.600 & 6.5 & 7.17 & 7.080 & 7.277 \\
$3 \mathrm{~s}$ & - & 3.042 & - & - & - & - \\
$3 \mathrm{p}_{0}$ & - & 3.211 & 3.2 & 3.60 & 3.442 & 3.677 \\
$3 \mathrm{p}_{ \pm 1}$ & - & 2.933 & 2.9 & 3.20 & 3.147 & 3.241 \\
$3 \mathrm{~d}_{0}$ & - & 3.211 & - & - & - & - \\
$3 \mathrm{~d}_{ \pm 1}$ & - & 3.113 & 3.0 & - & - & - \\
$3 \mathrm{~d}_{ \pm 2}$ & - & 2.889 & 2.9 & - & - & - \\
\hline
\end{tabular}

\subsection{The electric effect: $\boldsymbol{E} \perp \mathrm{c}$ axis and in $x$ direction}

In this case the perturbed Hamiltonian can be expressed as

$$
H^{\prime}(\lambda)=\frac{e^{2}}{\varepsilon_{0} r}\left(\lambda-\frac{1}{\left(1-\Delta^{2} \cos ^{2} \theta\right)^{1 / 2}}\right)-e E x
$$

and the matrix elements of $-e E x$ for $n=n^{\prime}$ can be evaluated as

$$
\begin{aligned}
\left\langle n^{\prime} l^{\prime} m^{\prime}|x| n \mid m\right\rangle & =\frac{3 n}{4 \lambda} a_{0}^{*}\left\{[ n ^ { 2 } - ( l + 1 ) ^ { 2 } ] ^ { 1 / 2 } \left[\left(\frac{(l+m+1)(l+m+2)}{(2 l+1)(2 l+3)}\right)^{1 / 2} \delta_{m^{\prime}, m+1}\right.\right. \\
& \left.-\left(\frac{(l-m+1)(l-m+2)}{(2 l+1)(2 l+3)}\right)^{1 / 2} \delta_{m^{\prime}, m-1}\right] \delta_{l^{\prime}, l+1} \\
& -\left[\left(\frac{(l-m)(l-m-1)}{(2 l-1)(2 l+1)}\right)^{1 / 2} \delta_{m^{\prime}, m+1}\right. \\
& \left.\left.-\left(\frac{(l+m)(l+m-1)}{(2 l-1)(2 l+1)}\right)^{1 / 2} \delta_{m^{\prime}, m-1}\right]\left(n^{2}-l^{2}\right)^{1 / 2} \delta_{l^{\prime}, l-1}\right\} .
\end{aligned}
$$

For the matrix elements with $n \neq n^{\prime}$, one can use equation (19b). The parameter $\lambda$ can be determined by PVM as mentioned in section 2.1 . 


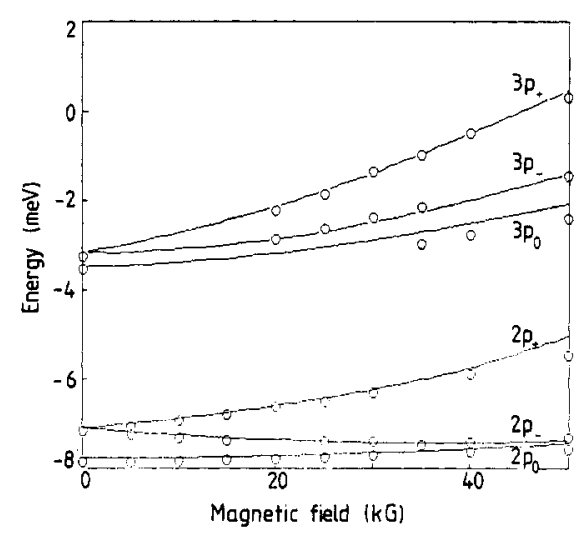

Figure 1. The binding energies of $2 p_{0, \pm 1}$ and $3 p_{0 . \pm 1}$ states in a magnetic field in the direction parallel to the $c$ axis. The observed data (circles) are adopted from Seiler et al (1982).

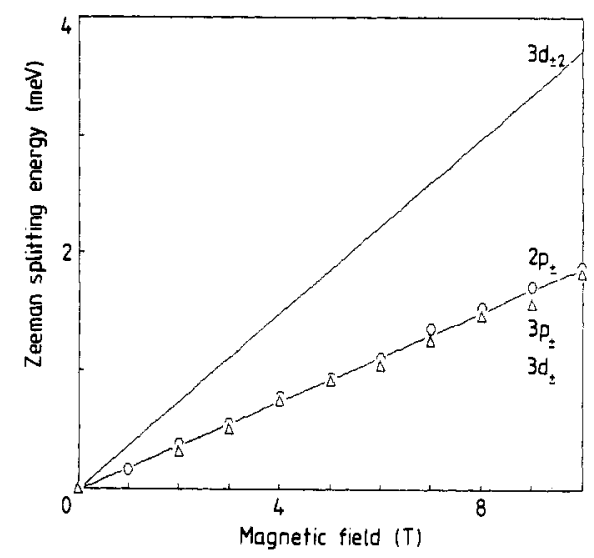

Figure 2. The Zeeman splittings of $2 \mathrm{p}_{ \pm 1}, 3 \mathrm{p}_{ \pm 1}$, $3 \mathrm{~d}_{ \pm 1}$ and $3 \mathrm{~d}_{ \pm 2}$ states in a magnetic field in the direction parallel to the $c$ axis. The observed data (circles for $2 \mathrm{p}_{ \pm 1}$ states and triangles for $3 \mathrm{p}_{ \pm 1}$ states) are adopted from Seiler et al (1982).

\section{Results and discussion}

The physical properties of CdS observed by Hopfield and Thomas (1961) and Seiler et al (1982) are presented in table 1. The binding energies calculated in the present work by using these physical properties are listed in table 2 . The observed and theoretical values obtained by Hopfield and Thomas (1961; denoted HT) and Seiler et al (1982; denoted SL) are also presented for comparison. Since the physical properties obtained in the previous work are slightly different from each other, to make a suitable comparison we present two sets of calculated results, which are obtained by using the physical properties of $\mathrm{HT}$ and SL respectively. We can see from table 2 that our results are in good agreement with the experimental values and are also consistent with the previous theoretical results in spite of the simplicity of our approach. However, some of our results are slightly higher than those of Seiler et al (1982), which are obtained by using a complex trial function; it is very difficult to extend their approach to the other higher excited states due to the difficulty in orthogonalising different excited-state wavefunctions.

The orbital magnetic effects on the binding energies due to a magnetic field applied in the direction parallel to the $c$ axis are shown as functions of magnetic field in figures $1-4$. Our results of $2 \mathrm{p}_{0, \pm 1}$ and $3 \mathrm{p}_{0, \pm 1}$ are compared with the observed data of Seiler et al (1982) in figure 1 . The calculated and observed Zeeman splitting and the diamagnetic effect of $2 \mathrm{p}_{0, \pm 1}$ and $3 \mathrm{p}_{0, \pm 1}$ are shown in figures 2 and 3 respectively. The observed Zeeman effect shown in figure 2 has been divided by a factor of 2 because the value of the $g$-factor chosen by Seiler et al is unreasonably large and is almost twice ours. In order to make a comparison, we also perform a conventional perturbation calculation of the energy splitting of $2 \mathrm{p}_{0, \pm 1}$ and $3 \mathrm{p}_{0, \pm 1}$ states due to a magnetic field applied in the direction parallel to the $c$ axis. The results are shown in figure 3 . One can note from figure 3 that the result by PVM obviously agrees better with the observed data of Seiler et al (1982) than do those of conventional perturbation calculation. The deviation between the conventional 

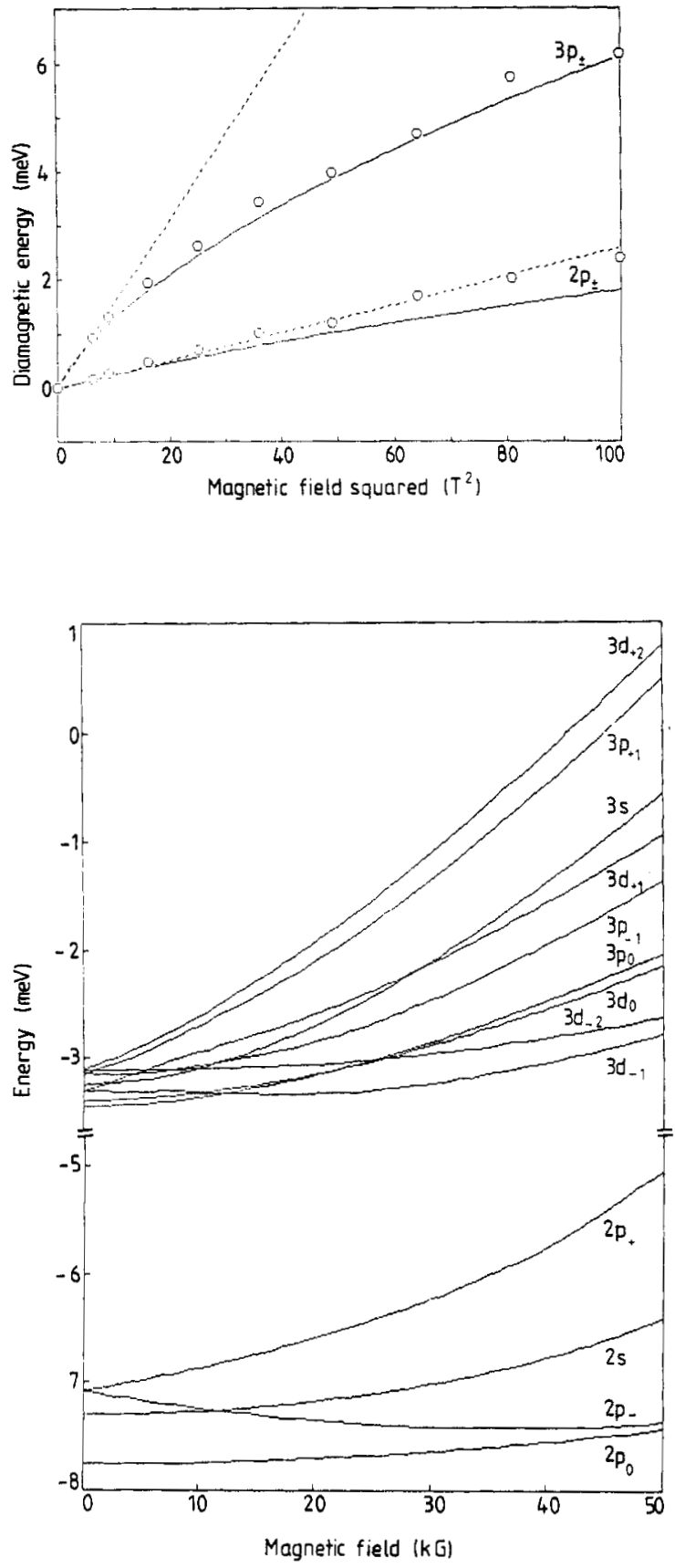

Figure 3. The diamagnetic shifts of $2 p_{ \pm 1}$ and $3 p_{ \pm 1}$ states in a magnetic field in the direction parallel to the $c$ axis. The observed data (circles) are adopted from Seiler et al (1982). The broken lines are obtained by a conventional perturbation treatment.
Figure 4. The binding energies of A-exciton states in a magnetic field in the direction parallel to the $c$ axis. The parameters are adopted from Seiler et al (1982).

perturbation calculation and the experimental data becomes quite large when the magnetic field strength is stronger. Figure 4 shows the binding energies of the A-exciton of $n=2$ and 3 states under a magnetic field in a direction parallel to the $c$ axis. In the calculation of figure 4 , the physical properties of CdS obtained by Seiler et al (1982) are used. 


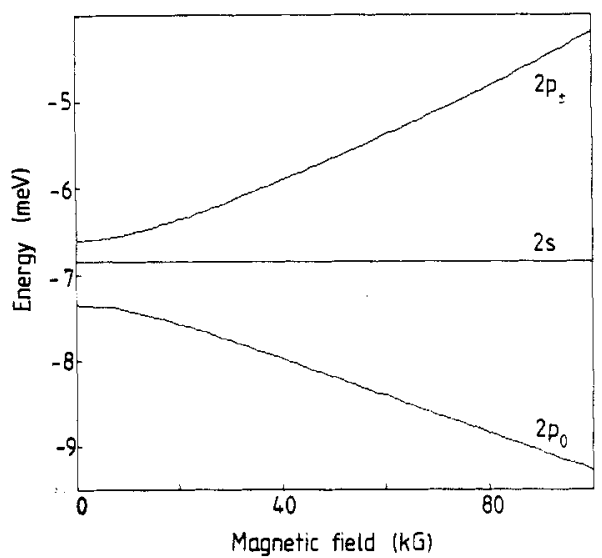

Figure 5. The energy splittings of the $n=2$ exciton states in a magnetic field perpendicular to the $c$ axis. The parameters are adopted from the work of Hopfield and Thomas (1961).

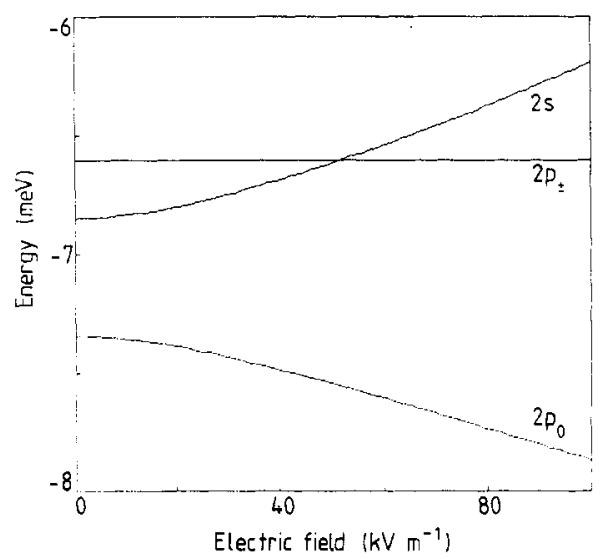

Figure 7. The energy shift of $n=2$ states in an electric field parallel to the $c$ axis. The parameters are adopted from the work of Hopfield and Thomas (1961).

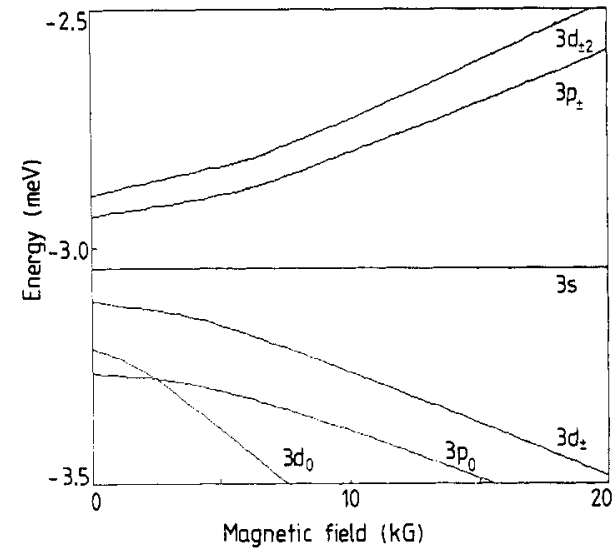

Figure 6. The energy splittings of the $n=3$ exciton states in a magnetic field perpendicular to the $c$ axis. The parameters are adopted from the work of Hopfield and Thomas (1961).

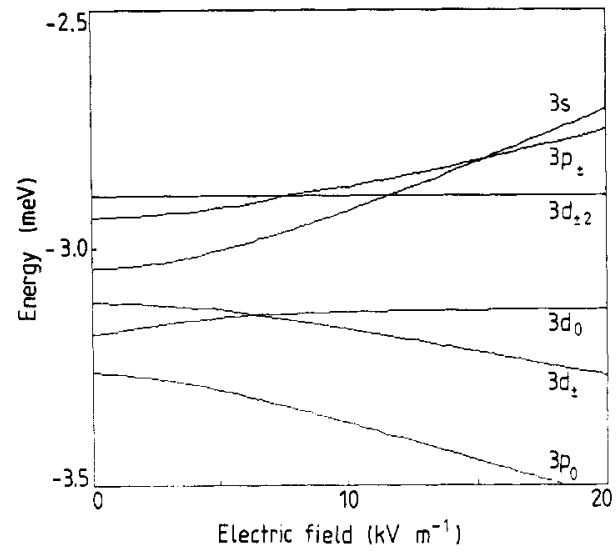

Figure 8. The energy shift of $n=3$ states in an electric field parallel to the $c$ axis. The parameters are adopted from the work of Hopfield and Thomas (1961).

Figures 5 and 6 show the binding energies of the A-exciton of $n=2$ and 3 states under a magnetic field applied in the direction perpendicular to the $c$ axis and pointed in the $x$ direction. The parameters used in our calculations of figures 5 and 6 are adopted from the observed data of Thomas and Hopfield $(1959,1961)$ and Hopfield and Thomas (1961). Since there are no experimental data available for these cases, we shall present our results for future publication.

The electric-field effects on exciton states of $\mathrm{CdS}$ are calculated by using the observed data of Hopfield and Thomas (1961). Figures 7 and 8 show the Stark splitting of $n=2$ and 3 states under an electric field applied in the direction parallel to the $c$ axis. The Stark shifts of $n=2$ and $n=3$ states under an electric field applied in the direction 


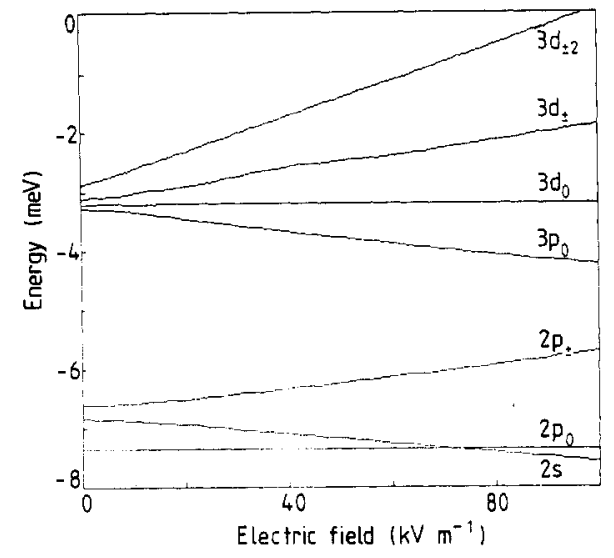

Figure 9. The energy shift of $n=2$ and $n=3$ states in an electric field perpendicular to the $c$ axis. The parameters are adopted from the work of Hopfield and Thomas (1961).

perpendicular to the $c$ axis and along the $x$ axis are shown in figure 9. We also perform a conventional perturbation calculation of the electric-field effects on the A-exciton states in CdS. It is found that the electric-field effects obtained from conventional perturbation treatment (not shown here) agree closely with those obtained by PVM when the electric field strength is weak. When the electric field strength becomes stronger, the deviation between PVM results and those obtained by the conventional perturbation method (not shown here) becomes slightly larger, but still falls within a reasonable range.

\section{Conclusions and summary}

We have calculated the binding energies, the Zeeman and Stark splittings of the states of CdS. The magnetic field and the electric field are assumed to be applied either in the direction of the $c$ axis or in the direction perpendicular to the $c$ axis. An approximation method that combines the spirit of both the conventional perturbation method and the variational principle is employed in our calculations. Our calculated results are used to compare the available experimental data and previous theoretical results obtained by conventional perturbation methods. The conventional variational calculation with a complex trial wavefunction (Pollmann 1976, Gerlach and Pollmann 1977, Seiler et al 1982, Grundmann and Bimberg 1988) is also capable of obtaining good agreement with the observed binding energies; however, in the variational approach the calculation must be started from the ground state. The excited states cannot be calculated independently and directly. Furthermore, the difficulty of seeking the orthogonal trial excited states increases with increasing $n$. On the contrary, our treatment can deal with any excited state independently and work them out very easily, and the approximation solution can be optimised by adjusting the parameter $\lambda$. Our method is certainly much easier and more efficient than the ordinary perturbation method. This is simply because, in the ordinary perturbation approach, one has to calculate a large number of matrix elements as one proceeds to calculate the higher-order corrections and there is no way to make the perturbation series converge as rapidly as possible. 


\section{Acknowledgment}

This work is partially supported by the National Science Council of the Republic of China under Grant Number NSC79-0208-M009-12.

\section{Appendix 1}

The values of $S_{l m}$ are given by

$$
S_{l m}=\left\langle l m\left|\frac{1}{\left(1-\Delta^{2} \cos ^{2} \theta\right)^{1 / 2}}\right| \operatorname{lm}\right\rangle
$$

Obviously $S_{l m}=S_{l,-m}$, because $Y_{l,-m}(\theta, \varphi)=(-1)^{m} Y_{l m}^{*}(\theta, \varphi)$. If we put

$$
I_{1}(\Delta)=\left(\sin ^{-1} \Delta\right) / \Delta \quad I_{2}(\Delta)=\left(1-\Delta^{2}\right)^{1 / 2} / \Delta^{2}
$$

we get by elementary integration

$$
\begin{aligned}
& S_{00}=I_{1}(\Delta) \\
& S_{10}=(3 / 2)\left[I_{1}(\Delta) / \Delta^{2}-I_{2}(\Delta)\right] \\
& S_{1, \pm 1}=(3 / 4)\left[\left(2+1 / \Delta^{2}\right) I_{1}(\Delta)-I_{2}(\Delta)\right] \\
& S_{2, \pm 0}=(5 / 8)\left\{\left[2-6 / \Delta^{2}+27 /\left(4 \Delta^{4}\right)\right] I_{1}(\Delta)+\left[3 / 2-27 /\left(4 \Delta^{2}\right)\right] I_{2}(\Delta)\right\} \\
& S_{2, \pm 1}=(15 / 4)\left\{\left[1 / \Delta^{2}-3 /\left(4 \Delta^{4}\right)\right] I_{1}(\Delta)-\left[1 / 2-3 /\left(4 \Delta^{2}\right)\right] I_{2}(\Delta)\right\} \\
& S_{2, \pm 2}=(15 / 16)\left\{\left[2-1 / \Delta^{2}+3 /\left(4 \Delta^{4}\right)\right] I_{1}(\Delta)+\left[3 / 2-3 /\left(4 \Delta^{2}\right)\right] I_{2}(\Delta)\right\} \\
& S_{30}=(7 / 4)\left\{\left[9 /\left(2 \Delta^{2}\right)-45 /\left(4 \Delta^{4}\right)+125 /\left(16 \Delta^{6}\right)\right] I_{1}(\Delta)-\left[7 / 6-145 /\left(24 \Delta^{2}\right)\right.\right. \\
& \left.\left.\quad+125 /\left(16 \Delta^{4}\right)\right] I_{2}(\Delta)\right\} \\
& \left.\left.\quad+5 /\left(16 \Delta^{4}\right)\right] I_{2}(\Delta)\right\} .
\end{aligned}
$$

Also

$$
S_{l^{\prime}, m}=\left\langle l^{\prime} m\left|\frac{1}{\left(1-\Delta^{2} \cos ^{2} \theta\right)^{1 / 2}}\right| l m\right\rangle
$$

so that

$$
S_{20,0}=(\sqrt{5} / 4)\left[\left(-2+3 / \Delta^{2}\right) I_{1}(\Delta)-3 I_{2}(\Delta)\right]
$$

\section{Appendix 2}

The non-zero off-diagonal matrix elements of the radial integrals are

$$
\begin{aligned}
& \left\langle R_{10}\left|e^{2} / \varepsilon_{0} r\right| R_{20}\right\rangle=(2 / 3)^{3} \sqrt{2} \lambda \\
& \left\langle R_{10}\left|e^{2} / \varepsilon_{0} r\right| R_{30}\right\rangle=(\sqrt{3} / 2) \lambda \\
& \left\langle R_{20}\left|e^{2} / \varepsilon_{0} r\right| R_{30}\right\rangle=-2(2 / 5)^{5} \sqrt{6} \lambda \\
& \left\langle R_{21}\left|e^{2} / \varepsilon_{0} r\right| R_{31}\right\rangle=12(2 / 5)^{5} \lambda
\end{aligned}
$$




$$
\begin{aligned}
& \left\langle R_{20}\left|e^{2} / \varepsilon_{0} r\right| R_{32}\right\rangle=-28(2 / 5)^{5} \sqrt{3 / 5} \lambda \\
& \left\langle R_{32}\left|e^{2} / \varepsilon_{0} r\right| R_{30}\right\rangle=\left[2 /\left(3^{4} \sqrt{10}\right)\right] \lambda \\
& \left\langle R_{32}\left|e^{2} / \varepsilon_{0} r\right| R_{10}\right\rangle=\left[1 /\left(3^{4} 2^{3} \sqrt{30}\right)\right] \lambda
\end{aligned}
$$

\section{References}

Blattnor G, Kurtze G, Schmieder G and Klingshirn C 1982 Phys. Rev. B 257413

Broser I and Rosenzweig M 1980 Phys. Rev. B 222000

Cho K, Suga S, Dreybrodt W and Willmann F 1975 Phys. Rev. B 111512

Damen T C, Nquyen V T and Gornik E 1977 Solid State Commun. 24179

Gerlach B and Pollmann J 1977 Nuovo Cim. B 38423

Grundmann M and Bimberg D 1988 Phys. Rev. B 3813486

Hopfield J J and Thomas D G 1961 Phys. Rev. 12235

Huang W J, Chou W C, Chuu D C, Han C S and Mei W N 1988 Semicond. Sci. Technol. 3202

Jiang T F and Shan Y 1985 J. Phys. C: Solid State Phys. 183399

Knox R S 1963 Solid State Physics (New York: Academic) p 76

Kohn W and Luttinger J M 1955 Phys. Rev. $971721 ; 98915$

Lee Y C, Mei W N and Liu K C 1982 J. Phys. C: Solid State Phys. 15 L469

Lipari N O 1972 Nuovo Cim. 8B 406

Litton C W, Reynolds D C and Collins T C 1972 Phys. Rev. B 62269

Nquyen V T, Damen T C and Gornik E 1977a Appl. Phys. Lett. 3033

Nquyen V T, Damen T C, Gornik E and Patel C K N 1977b Appl. Phys. 31603

Peyghambarian N, Koch S W, Lindberg M, Fluegel B and Toffre M 1989 Phys. Rev. Lett. 621185

Pollmann J 1976 Solid State Commun, 19361

Seiler D G, Heiman D, Feigenblatt R, Aggarwal R L and Lax B 1982 Phys. Rev. B 257666

Seiler D G, Heiman D and Wherertt B S 1983 Phys. Rev. B 272355

Shad J and Damen T C 1971 Solid State Commun. 91285

Stafford R G and Sondergeld M 1974 Phys. Rev. B 103471

Suga S, Bettini M, Dreybrodt W and Cho K 1975 Coll. Int. Centre Nat. Rech. Sci. 242217

Thomas D G and Hopfield J J 1959 Phys. Rev. 116573 1961 Phys. Rev. 124657

Venghaus H, Suga S and Cho K 1977 Phys. Rev. B 164419

Voigt J, Spiegelberg F and Senoner M 1979 Phys. Status Solidi b 91184 